\title{
THE LAWS OF SOME METABELIAN VARIETIES
}

\author{
RICHARD LEVINGSTON \\ (Received 22 April 1980, Revised 10 July 1980) \\ Communicated by D. E. Taylor
}

\begin{abstract}
It is shown that if $m, n$ are relatively prime positive integers, then the variety consisting of those soluble groups of exponent $m n$ in which any subgroup of exponent $m$ or $n$ is abelian has a basis of two-variable laws.
\end{abstract}

1980 Mathematics subject classification (Amer. Math. Soc.): 20 E 10.

Since the paper of Higman (1959), it has been of interest to ask which varieties have a 2-variable basis for their laws. In this note, we show that certain metabelian varieties are defined by 2 -variable laws.For unexplained results and notation on varieties of groups see Neumann (1967), while for other group-theoretical results see Gorenstein (1968).

THEOREM. Let $m$ and $n$ be relatively prime positive integers. Then the following set of laws forms a basis for the laws of the variety $\mathfrak{A}_{m} \mathfrak{A}_{n} \vee \mathfrak{A}_{n} \mathfrak{A}_{m}$ :

(1) $x^{m n}=1$.

(2) $\left[x^{m}, y^{m}\right]^{m}=1$.

(3) $\left[x^{n}, y^{n}\right]^{n}=1$.

(4) $\left[[x, y],\left[x^{-1}, y\right]\right]=1$.

Let $\mathfrak{B}$ denote the variety defined by the laws (1)-(4), and let $\mathfrak{U}$ denote the variety $\mathfrak{A}_{m} \mathfrak{A}_{n} \vee \mathfrak{A}_{n} \mathfrak{A}_{m}$. We prove that $\mathfrak{U}=\mathfrak{B}$ in a series of lemmas. Note however that the laws (1)-(4) hold in $\mathfrak{A}_{m} \mathfrak{A}_{n}$ and in $\mathfrak{A}_{n} \mathfrak{A}_{m}$, so we have $\mathfrak{U}<\mathfrak{B}$.

LEMMA 1. (a) Groups in $\mathfrak{B}$ of exponent dividing $m$ or $n$ are abelian.

(b) Finitely-generated soluble groups in $\mathfrak{B}$ are in $\mathfrak{U}$.

(c) 2-generator groups in $\mathfrak{B}$ are metabelian.

( Copyright Australian Mathematical Society 1981 
Proof. The law (3) reduces to $[x, y]=1$ in a group of exponent dividing $m$, as does the law (2) in a group of exponent dividing $n$. Hence (a) holds.

Let $G \in \mathfrak{B}$ be a finitely-generated soluble group. Then $G$ is finite. Now $F(G)=F_{1} \times F_{2}$, where $F_{1}$ has exponent dividing $m$ and $F_{2}$ has exponent dividing $n$. Let $G_{i}=G / F_{i}$ for $i=1,2$. Then $G$ is a subgroup of $G_{1} \times G_{2}$, and it suffices to show that $G_{1}$ and $G_{2}$ lie in $\mathfrak{U}$.

Now $F\left(G_{1}\right)$ has exponent dividing $n$, by law (1) and part (a). But if $g \in G_{1}$ has order dividing $n$ then again by law (1) $\left\langle g, F\left(G_{1}\right)\right\rangle$ has exponent dividing $n$, and so by (a) is abelian. Hence every element of $G_{1}$ of order dividing $n$ centralizes $F\left(G_{1}\right)$. But $e_{G}\left(F\left(G_{1}\right)\right) \leqslant F\left(G_{1}\right)$ (Gorenstein (1968), Theorem 6.1.3), so $F\left(G_{1}\right)$ contains all the elements of $G_{1}$ of order dividing $n$. Hence $G_{1} / F\left(G_{1}\right)$ has exponent dividing $m$, and so by part (a) is abelian. Then $G_{1} \in \mathfrak{A}_{n} \mathfrak{A}_{m}<\mathfrak{U}$. An exactly similar argument shows that $G_{2} \in \mathfrak{U}_{m} \mathfrak{A}_{n} \leqslant \mathfrak{U}$. Hence $G \in \mathfrak{U}$.

By Theorem 2.1 of Higman (1959), (c) is a consequence of the law (4).

Lemma 2. $\left[x^{m},\left(x^{n}\right)^{y}\right]=1$ is a law of $\mathfrak{B}$.

ProOF. First we show that $\left[x^{m},\left(x^{n}\right)^{y}\right]=1$ is a law of $\mathfrak{U}$. In other words, we must show that it is a law in $\mathfrak{U}_{m} \mathfrak{A}_{n}$ and in $\mathfrak{A}_{n} \mathfrak{U}_{m}$. In $\mathfrak{A}_{m} \mathfrak{A}_{n}$, a commutator $c$ has order $m$, and so since $(m, n)=1, c$ is an $n$th power. Also $n$th powers commute. So $\left[x, y, z^{n}\right]=1$ is a law of $\mathfrak{A}_{m} \mathfrak{A}_{n}$. But $\left[x^{m},\left(x^{n}\right)^{y}\right]=\left[x^{m}, y^{-1}, x^{n}\right\}^{y}$, so $\left[x^{m},\left(x^{n}\right)^{y}\right]=1$ is a law of $\mathfrak{A}_{m} \mathfrak{A}_{n}$.

Similarly $\left[z^{m},[x, y]\right]=1$ is a law of $\mathfrak{A}_{n} \mathfrak{A}_{m}$. But $\left[x^{m},\left(x^{n}\right)^{y}\right]=\left[x^{m},\left[x^{n}, y\right]\right]$, so $\left[x^{m},\left(x^{n}\right)^{y}\right]=1$ is a law of $\mathfrak{A}_{n} \mathfrak{A}_{m}$. Hence $\left[x^{m},\left(x^{n}\right)^{y}\right]=1$ is a law of $\mathfrak{U}$.

But now suppose $G \in \mathfrak{B}$ does not satisfy $\left[x^{m},\left(x^{n}\right)^{y}\right]=1$. Then $G$ contains elements $g, h$ with $\left[g^{m},\left(g^{n}\right)^{h}\right] \neq 1$. But by Lemma 1 (b) and (c) $\langle g, h\rangle \in \mathbb{U}$. Hence $\left[g^{m},\left(g^{n}\right)^{h}\right]=1$, a contradiction. Hence $\left[x^{m},\left(x^{n}\right)^{y}\right]=1$ is a law of $\mathfrak{B}$.

LEMMA 3. $\mathfrak{B}$ contains no non-abelian simple group.

Proof. Suppose $G \in \mathfrak{B}, G$ a non-abelian simple group. Then we deduce some properties of $G$.

(i) If $g \in G$, then $g^{m}=1$ or $g^{n}=1$.

For if $g \in G$ with $g^{m} \neq 1$ and $g^{n} \neq 1$, then by Lemma $2, \bigodot_{G}\left(g^{m}\right)$ contains all conjugates of $g^{n}$. But $G$ is simple, so $G$ is generated by the conjugates of $g^{n}$. Then $g^{m}$ is central in $G$, which is absurd, since $G$ is a non-abelian simple group.

(ii) Suppose that $g, h$ are non-commuting elements of $G$ of the same order, and let $H=\langle g, h\rangle$. Then $H$ is a Frobenius group, with $\langle g\rangle$ and $\langle h\rangle$ as Frobenius complements. In particular, there is an integer $\alpha$ with $\langle g\rangle=\left\langle g^{\alpha}\right\rangle$ and $g^{-\alpha} h \in H^{\prime}$. 
Let $g, h$ have order $\mu$. By (i), we may assume for definiteness, that $\mu$ divides $m$. Then by Lemma 1 (c), $H$ is metabelian, and so finite. Again $g$ and $h$ are $n$th powers. But $H^{\prime}$ is generated by elements $[a, b]$ with a conjugate of $g$ and $b$ a conjugate of $h$. Then by law (3), these elements have order dividing $n$. Since $H^{\prime}$ is abelian, it follows that $H^{\prime}$ has exponent dividing $n$. Now by (i), $H / H^{\prime}$ acts regularly on $H^{\prime}$. Hence $H$ is a Frobenius group. Since $H / H^{\prime}$ is abelian, it is cyclic (Gorenstein (1968), Theorem 5.3.14(ii). See also Theorem 10.3.1).

Now by (i), $H^{\prime} \cap\langle g\rangle=H^{\prime} \cap\langle h\rangle=1$, as $\left(\mu,\left|H^{\prime}\right|\right)=1$. But $H / H^{\prime}=$ $\left\langle g H^{\prime}, h H^{\prime}\right\rangle$, so $H / H^{\prime}$ has exponent exactly $\mu$. Since $H / H^{\prime}$ is cyclic, we have $\left|\boldsymbol{H}: H^{\prime}\right|=\mu$, whence $\boldsymbol{H}=H^{\prime}\langle g\rangle=H^{\prime}\langle h\rangle$. In other words, $\langle g\rangle$ and $\langle h\rangle$ are Frobenius complements. Then $\langle g\rangle$ and $\langle h\rangle$ are conjugate in $H$ (Gorenstein (1968), Theorem 6.2.1(ii)). Choose $a \in H$ with $\left\langle h^{a}\right\rangle=\langle g\rangle$, say $h^{a}=g^{\alpha}$. Then $g^{-\alpha} h=\left(h^{a}\right)^{-1} h=[a, h] \in H^{\prime}$ as required.

(iii) $G$ contains a non-cyclic abelian subgroup.

Let $p$ be the largest prime dividing the exponent of $G$. Then $G$ is generated by elements of order $p$. Hence $G$ contains a pair $g, h$ of non-commuting elements of order $p$. Let $H=\langle g, h\rangle$. Then by (ii) $H$ is a Frobenius group, with $H^{\prime}$ abelian. Let $C$ be a complement to $H^{\prime}$ in $H$, and let $q$ be a prime dividing $\left|H^{\prime}\right|$. Then $|C|=p$, and $C$ acts regularly on the abelian group $O_{q}\left(H^{\prime}\right)$. Since $q<p, O_{q}\left(H^{\prime}\right)$ must be non-cyclic.

(iv) $G$ does not exist.

By (iii), $G$ contains a non-cyclic abelian subgroup, so for some prime $p, G$ contains the non-cyclic group of order $p^{2}$. Hence choose $g, h \in G$ such that $\langle g, h\rangle$ is non-cyclic of order $p^{2}$. Suppose for definiteness that $p$ divides $m$.

Let $A=\mathcal{C}_{G}(g)$. By (i) $A$ has exponent dividing $m$, so by Lemma 1 (a) $A$ is abelian. If $a \in A$ then again $\mathcal{C}_{G}(a)$ has exponent dividing $m$, and is abelian. Also $A \leqslant \bigcup_{G}(a)$. So $\mathcal{C}_{G}(a)$ centralizes $g$. Then $\bigcup_{G}(a) \leqslant A$. Hence we have $A=\mathcal{C}_{G}(a)$ for any $a \in A^{\#}$. In particular $A=\mathcal{C}_{G}\left(g^{-1} h\right)$.

Now as $G$ is simple, $G$ is generated by elements of order $p$ (for example, the conjugates of $g$ ). Then there is an element $k$ of order $p$ in $G-A$, as $A$ is abelian but $G$ is not. Let $H_{1}=\langle g, k\rangle$ and $H_{2}=\langle h, k\rangle$. Then by (ii) there are integers $\alpha, \beta$ with $1 \leqslant \alpha, \beta<p$ such that $g^{-\alpha} k \in H_{1}^{\prime}$ and $h^{-\beta} k \in H_{2}^{\prime}$. Replacing $g$ by $g^{\alpha}$ and $h$ by $h^{\beta}$, we suppose that $g^{-1} k \in H_{1}^{\prime}$ and $h^{-1} k \in H_{2}^{\prime}$. But $H_{1}^{\prime}$ and $H_{2}^{\prime}$ have exponent dividing $n$, so $\left(g^{-1} k\right)^{n}=\left(h^{-1} k\right)^{n}=1$. Then $g^{-1} k, h^{-1} k$ are $m$ th powers, and so by law (2), $\left[g^{-1} k, k^{-1} h\right]^{m}=1$. But since $[g, h]=1,\left[g^{-1} k, k^{-1} h\right]=$ $\left[k, g^{-1} h\right]$. As $k^{m}=\left(g^{-1} h\right)^{m}=1,\left[g^{-1} k, k^{-1} h\right]^{n}=\left[k, g^{-1} h\right]^{n}=1$, by law (3). Since $(m, n)=1$, we have $\left[k, g^{-1} h\right]=1$. Then $k \in \mathcal{C}_{G}\left(g^{-1} h\right)=A$, contradicting the choice of $k$.

LEMMA 4. $\mathfrak{U}=\mathfrak{B}$. 
Proof. Suppose $\mathfrak{U} \neq \mathfrak{B}$. Then as $\mathfrak{U}<\mathfrak{B}$, there is a law of $\mathfrak{U}$ which is not a law of $\mathfrak{B}$. Hence there is a finitely-generated group $G$ with $G \in \mathfrak{B}-\mathfrak{u}$. By Lemma 3 and Lemma 1 (b), all finite groups in $\mathfrak{B}$ are in $\mathcal{U}$. Hence $G$ is infinite. We show first that $G^{\prime \prime}$ is perfect. Since $G / G^{\prime \prime \prime}$ is finitely-generated and soluble, we have by Lemma 1 (b) that $G / G^{\prime \prime \prime} \in \mathfrak{U}$. But all groups in $\mathfrak{U}$ are metabelian. Hence $G^{\prime \prime}=G^{\prime \prime \prime}$ as required.

Now $G / G^{\prime \prime}$ is finite, while $G$ is finitely-generated. Then $G^{\prime \prime}$ is finitely-generated. Now by Zorn's Lemma, $G^{\prime \prime}$ has a maximal normal subgroup $N$. Then $G^{\prime \prime} / N$ is a simple group, which is non-abelian since $G^{\prime \prime}$ is perfect. But $G^{\prime \prime} / N \in$ $\mathfrak{B}$, contradicting Lemma 3 and completing the proof.

\section{References}

D. Gorenstein (1968), Finite groups (Harper and Row, New York, Evanston, London).

G. Higman (1959), 'Some remarks on varieties of groups', Quart.J. Math. Oxford Ser. 10, 165-178.

H. Neumann (1967), Varieties of groups (Ergebnisse der Mathematik und ihrer Grenzgebiete 37, Springer-Verlag, Berlin, Heidelberg, New York).

Department of Mathematics

Institute of Advanced Studies

The Australian National University

P.O. Box 4

Canberra, A.C.T. 2600

Australia 